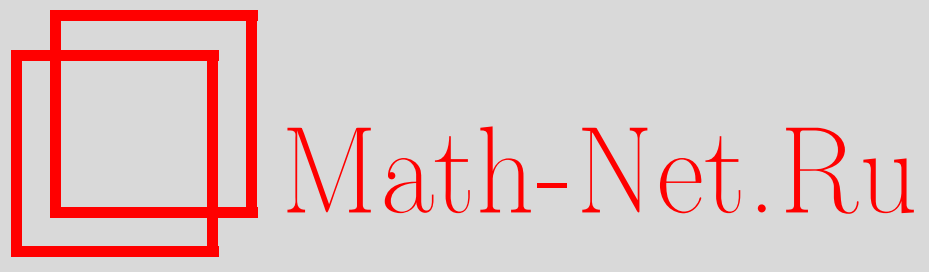

А. О. Радомский, О возможности усиления неравенств типа Сидона, Матем. заметки, 2013, том 94, выпуск $5,792-795$

DOI: https://doi.org/10.4213/mzm10335

Использование Общероссийского математического портала Math-Net.Ru подразумевает, что вы прочитали и согласны с пользовательским соглашением http://www . mathnet.ru/rus/agreement

Параметры загрузки:

IP : 54.197 .130 .99

26 апреля 2023 г., 04:39:56

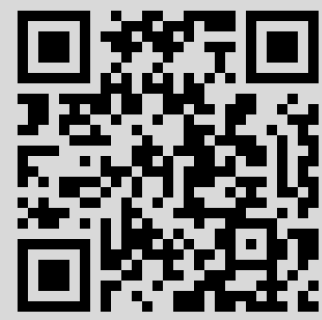




\section{О возможности усиления неравенств типа Сидона}

\section{А. О. Радомский}

Обобщения классического неравенства Сидона (см., например, [1; т. 1, с. 393-395]) были установлены в работах [2]-[4]. Следующая теорема является усилением теоремы 2.3 из работы [2] и теоремы 1 из работы [4].

Теорема 1. Пусть последовательность натуральных чисел $\left\{n_{k}\right\}_{k=1}^{\infty}$ удовлетворлет условию $n_{k+1} / n_{k} \geqslant \lambda>1, k=1,2, \ldots$. Существуют константы $c=c(\lambda)>0 u \gamma=\gamma(\lambda) \in$ $(0,1)$, зависящие лишь от $\lambda$, такие, что для любого тригонометрического полинома вида

$$
f(x)=\sum_{k=l}^{N} p_{k}(x) \cos n_{k} x,
$$

где $p_{k}$ - вещественные тригонометрические полиномы $c \operatorname{deg}\left(p_{k}\right) \leqslant\left[\gamma n_{l}\right], k=l, \ldots, N$, $N \geqslant l, l=1,2, \ldots$, справедливо неравенство

$$
\|f\|_{\infty} \geqslant c \sum_{k=l}^{N}\left\|p_{k}\right\|_{1} .
$$

Здесъ $\|\cdot\|_{p}-$ норма в пространстве $L^{p}(0,2 \pi), 1 \leqslant p \leqslant \infty$ :

$$
\|f\|_{\infty}=\operatorname{ess} \sup _{[0,2 \pi]}|f(x)|, \quad\|f\|_{p}=\left(\frac{1}{2 \pi} \int_{0}^{2 \pi}|f(x)|^{p} d x\right)^{1 / p}, \quad 1 \leqslant p<\infty .
$$

Приведенный ниже результат показывает, что в теореме 1 (при $n_{k}=2^{k}$ ) условие $\operatorname{deg} p_{k} \leqslant$ $\left[\gamma 2^{l}\right]$ нельзя заменить на $\operatorname{deg} p_{k} \leqslant\left[2^{k-k^{\varepsilon}}\right]$ ни при каком $\varepsilon \in(0,1)$. Доказательство теоремы 2 опирается на идеи из работы [5].

Теорема 2. Пусть $\varepsilon, \widetilde{\varepsilon}-$ действителъные числа, причем $1 / 2 \leqslant \varepsilon<\widetilde{\varepsilon}<1$. Для любого $W \in \mathbb{N}$ существуют вещественные тригонометрические полиномы $p_{k}(x), k=1, \ldots, W$, maкue, что $\operatorname{deg} p_{k} \leqslant\left[2^{k-k^{\varepsilon}}\right],\left\|p_{k}\right\|_{1} \geqslant 1 / 3,\left\|p_{k}\right\|_{\infty} \leqslant 70, k=1, \ldots, W, u$

$$
\max _{1 \leqslant n \leqslant W}\left\|\sum_{k=1}^{n} p_{k}(x) \cos 2^{k} x\right\|_{\infty} \leqslant C W^{\widetilde{\varepsilon}},
$$

где $C=C(\varepsilon, \widetilde{\varepsilon})>0-$ константа, зависящая лищь от $\varepsilon$ и $\widetilde{\varepsilon}$.

ДокАЗАТЕЛЬСТво теОРемы 2. Возьмем $k_{0}=k_{0}(\varepsilon, \widetilde{\varepsilon}) \in \mathbb{N}, k_{0}>5$, такое, что при $k \geqslant k_{0}$ выполнено:

1) $2^{k-k^{\varepsilon}} \geqslant 6$

2) $2^{k^{\varepsilon}-k^{\widetilde{\varepsilon}}} k^{3}<1 / 4$

3) $2^{(k-1)^{\varepsilon}-k^{\varepsilon}}>1 / 2$;

4) $k^{\widetilde{\varepsilon}}<(1 / 8) k$.

Обозначим $m_{k}:=\left[2^{k-k^{\varepsilon}}\right]$. Пусть функция $f \in L^{2}(0,2 \pi)$ и последовательность $\left\{l_{k}\right\}_{k=1}^{\infty}$, $l_{k} \in \mathbb{N}$, удовлетворяет условию

$$
\frac{l_{k+1}}{l_{k}} \geqslant q>1, \quad k=1,2, \ldots
$$

DOI: $10.4213 / \mathrm{mzm} 10335$

(C) А. О. РАдомский, 2013 
Тогда для $S^{*}(x)=\sup _{k \geqslant 1}\left|S_{l_{k}}(f, x)\right|$ справедливо неравенство (см., например, [1; т. 2, с. 246])

$$
\left\|S^{*}\right\|_{2} \leqslant c_{0}(q)\|f\|_{2},
$$

где константа $c_{0}=c_{0}(q)>0$ зависит только от $q$. Возьмем константу $c_{0}$ из неравенства (2), соответствующую $q=23 / 16$. Положим $\alpha=\max \left(70 \sqrt{8} c_{0}, 1\right)$. Возьмем произвольное $W \geqslant 8 k_{0}, W \in \mathbb{N}$. Положим $a=\left[W^{\widetilde{\varepsilon}}\right]$. Полиномы $p_{k}(x), k=1, \ldots, W$, удовлетворяющие условиям теоремы, будем строить по индукции. Положим

$$
p_{k}(x) \equiv 1, \quad k=1, \ldots, k_{0}+a .
$$

Пусть полиномы $p_{1}(x), \ldots, p_{N}(x)\left(N \geqslant k_{0}+a\right)$ уже построены. Построим $p_{N+1}(x)$. Положим

$$
F_{k}(x):=\sum_{j=k_{0}}^{k} p_{j}(x) \cos 2^{j} x
$$

$\delta_{j}(x):=p_{j}(x) \cos 2^{j} x$ и

$$
\begin{gathered}
E_{N}^{k}:=\left\{x \in[0,2 \pi):\left|F_{k}(x)\right|>\alpha \sqrt{N}\right\}, \quad k=k_{0}, \ldots, N, \\
B_{N}:=\bigcup_{k=k_{0}}^{N} E_{N}^{k}, \quad \widetilde{B}_{N}:=\bigcup_{k=k_{0}}^{N-a} O_{\left(2 \pi(N-k)^{2}\right) / m_{N}}\left(E_{N}^{k}\right),
\end{gathered}
$$

где $O_{\varepsilon}(G)$ обозначает $\varepsilon$-окрестность множества $G$ на $[0,2 \pi)$ с метрикой окружности. Через $d(x, y)$ будем обозначать расстояние на окружности $[0,2 \pi)$ между точками $x$ и $y$. Положим

$$
\begin{gathered}
\Lambda_{N+1}:=\left\{j \in\left\{1, \ldots, m_{N+1}\right\}: \widetilde{B}_{N} \cap O_{\pi / m_{N+1}}\left(\frac{2 \pi j}{m_{N+1}}\right)=\varnothing\right\}, \\
p_{N+1}(x):=\frac{1}{m_{N+1}} \sum_{j \in \Lambda_{N+1}} K_{m_{N+1}-1}\left(x-\frac{2 \pi j}{m_{N+1}}\right),
\end{gathered}
$$

где

$$
K_{m-1}(x)=\frac{\sin ^{2}(m x / 2)}{m \sin ^{2}(x / 2)}
$$

- ядра Фейера. Оценим $\left|\Lambda_{N+1}\right|$. Используя неравенство (2), получаем

$$
\mu\left(B_{N}\right) \leqslant \frac{c_{0}^{2} 2 \pi\left\|F_{N}\right\|_{2}^{2}}{\alpha^{2} N}=\frac{c_{0}^{2} 2 \pi \sum_{k=k_{0}}^{N}\left\|\delta_{k}\right\|_{2}^{2}}{\alpha^{2} N} \leqslant \frac{c_{0}^{2} 2 \pi \sum_{k=k_{0}}^{N}\left\|\delta_{k}\right\|_{\infty}^{2}}{\alpha^{2} N} \leqslant \frac{c_{0}^{2} 2 \pi 70^{2} N}{\alpha^{2} N} \leqslant \frac{\pi}{4} .
$$

Обозначим через $\operatorname{Conn} G$ число компонент связности множества $G$. Так как $\operatorname{deg}\left|F_{k}(x)\right|^{2} \leqslant$ $4 \cdot 2^{k}$, то получаем

$$
\text { Conn } E_{N}^{k} \leqslant 4 \cdot 2^{k}
$$

Следовательно,

$$
\text { Conn } \widetilde{B}_{N} \leqslant \sum_{k=k_{0}}^{N-a} \operatorname{Conn} E_{N}^{k} \leqslant 4 \frac{2^{N+1}}{2^{a}} \leqslant 4 \frac{2^{N+1}}{2^{W^{\widetilde{\varepsilon}}-1}} \leqslant 8 \frac{2^{N+1}}{2^{N^{\widetilde{\varepsilon}}}} .
$$

Учитывая $(7),(8)$ и условие 1$)$ на $k_{0}$, получаем (см. (4))

$$
\begin{aligned}
\mu\left(\widetilde{B}_{N}\right) & \leqslant \mu\left(B_{N}\right)+\sum_{k=k_{0}}^{N-a} \frac{4 \pi(N-k)^{2}}{m_{N}} \operatorname{Conn} E_{N}^{k} \leqslant \frac{\pi}{4}+16 \pi \sum_{k=k_{0}}^{N-a} \frac{(N-k)^{2}}{2^{N} / 2^{N^{\varepsilon}}-1} 2^{k} \\
& \leqslant \frac{\pi}{4}+16 \pi \sum_{k=k_{0}}^{N-a} \frac{(N-k)^{2}}{2^{N} / 2^{N^{\varepsilon}}-2^{N-1} / 2^{N^{\varepsilon}}} 2^{k}=\frac{\pi}{4}+32 \pi 2^{N^{\varepsilon}} \sum_{s=a}^{N-k_{0}} \frac{s^{2}}{2^{s}} .
\end{aligned}
$$


Поскольку функция $f(x)=x^{2} / 2^{x}$ монотонно убывает при $x \geqslant 3$ и, как нетрудно видеть, $a>5$, получаем (см. условия 2) и 4$)$ на $k_{0}$ )

$$
\mu\left(\widetilde{B}_{N}\right) \leqslant \frac{\pi}{4}+32 \pi 2^{N^{\varepsilon}} \frac{a^{2}}{2^{a}} N \leqslant \frac{\pi}{4}+\frac{\pi}{2} \frac{2^{W^{\varepsilon}} W^{3}}{2^{W^{\widetilde{\varepsilon}}-1}} \leqslant \frac{\pi}{4}+\frac{\pi}{4}=\frac{\pi}{2} .
$$

Оценим число интервалов

$$
I_{j}:=O_{\pi / m_{N+1}}\left(\frac{2 \pi j}{m_{N+1}}\right),
$$

которые пересекаются с $\widetilde{B}_{N}$. Для каждого такого интервала возможен один из двух случаев: либо он содержит граничную точку $\widetilde{B}_{N}$, либо он целиком содержится в $\widetilde{B}_{N}$. Число интервалов первого типа (см. (9)) не превосходит 2 Conn $\widetilde{B}_{N} \leqslant 16 \cdot 2^{N+1} / 2^{N^{\widetilde{\varepsilon}}}$. Пусть число интервалов второго типа равно $S$. Тогда, поскольку интервалы $I_{j}$ не пересекаются при разных $j$, то (см. (10)) имеем

и, следовательно,

$$
S \frac{2 \pi}{m_{N+1}} \leqslant \mu\left(\widetilde{B}_{N}\right) \leqslant \frac{\pi}{2}
$$

$$
S \leqslant \frac{m_{N+1}}{4} \leqslant \frac{1}{4} \frac{2^{N+1}}{2^{(N+1)^{\varepsilon}}} .
$$

Отсюда находим (см. условия 1)-3) на $k_{0}$ )

$$
\left|\Lambda_{N+1}\right| \geqslant \frac{2^{N+1}}{2^{(N+1)^{\varepsilon}}}\left(\frac{3}{4}-16 \frac{2^{(N+1)^{\varepsilon}}}{2^{N^{\widetilde{\varepsilon}}}}\right)-1 \geqslant \frac{1}{3} \frac{2^{N+1}}{2^{(N+1)^{\varepsilon}}} .
$$

Учитывая, что $K_{m}(x) \geqslant 0$ и $\left\|K_{m}\right\|_{1}=1$, имеем (см. (6), (11))

$$
\left\|p_{N+1}\right\|_{1}=\frac{1}{m_{N+1}} \sum_{j \in \Lambda_{N+1}}\left\|K_{m_{N+1}-1}\right\|_{1} \geqslant \frac{1}{m_{N+1}} \frac{1}{3} \frac{2^{N+1}}{2^{(N+1)^{\varepsilon}}} \geqslant \frac{1}{3} .
$$

Хорошо известна оценка

$$
K_{m-1}(x) \leqslant \begin{cases}10 m \cdot \min \left(1, \frac{1}{m^{2} x^{2}}\right), & |x| \leqslant \pi, \\ 10 m \cdot \min \left(1, \frac{1}{m^{2}(2 \pi-|x|)^{2}}\right), & \pi<|x| \leqslant 2 \pi .\end{cases}
$$

Из (12) нетрудно вывести (см. (6)), что $\left\|p_{N+1}\right\|_{\infty} \leqslant 70$. Оценим $\left\|F_{N+1}\right\|_{\infty}$. Положим

$$
k(x):=\max \left\{k_{0} \leqslant j \leqslant N+1:\left|F_{j}(x)\right| \leqslant \alpha \sqrt{N}\right\} .
$$

Поскольку

$$
\left|F_{k_{0}}(x)\right|=\left|p_{k_{0}}(x) \cos 2^{k_{0}} x\right|=\left|\cos 2^{k_{0}} x\right| \leqslant 1 \leqslant \alpha \leqslant \alpha \sqrt{N}
$$

то функция $k(x)$ корректно определена. Возьмем произвольное $x \in[0,2 \pi)$. Рассмотрим два случая:

1) $k(x) \geqslant N-a$; тогда

$$
\left|F_{N+1}(x)\right| \leqslant\left|F_{k(x)}(x)\right|+\sum_{j=k(x)+1}^{N+1}\left|\delta_{j}(x)\right| \leqslant \alpha \sqrt{N}+70(a+1) ;
$$

2) $k(x)<N-a$; тогда

$$
\begin{aligned}
\left|F_{N+1}(x)\right| & \leqslant\left|F_{k(x)}(x)\right|+\sum_{j=k(x)+1}^{k(x)+a+1}\left|\delta_{j}(x)\right|+\sum_{j=k(x)+a+2}^{N+1}\left|\delta_{j}(x)\right| \\
& \leqslant \alpha \sqrt{N}+70(a+1)+\sum_{j=k(x)+a+2}^{N+1}\left|\delta_{j}(x)\right| .
\end{aligned}
$$


Оценим последнее слагаемое. Покажем, что (см. (3), (4))

$$
x \in E_{j-1}^{k(x)+1} \subset \widetilde{B}_{j-1}, \quad j=k(x)+a+2, \ldots, N+1 .
$$

Действительно, $\left|F_{k(x)+1}(x)\right|>\alpha \sqrt{N} \geqslant \alpha \sqrt{j-1}$, и так как $k(x) \geqslant k_{0}$ и $j-1 \geqslant k(x)+1+a \geqslant$ $k_{0}+1+a$, то (15) выполняется. Следовательно (см. (5)), для любого $l \in \Lambda_{j}$

$$
d\left(x, \frac{2 \pi l}{m_{j}}\right) \geqslant \frac{2 \pi|j-1-(k(x)+1)|^{2}}{m_{j-1}}=\frac{2 \pi|j-k(x)-2|^{2}}{m_{j-1}} .
$$

Из (16) (см. также (6), (12) и условия 1) и 3) на $\left.k_{0}\right)$ нетрудно вывести, что $\left|\delta_{j}(x)\right| \leqslant 40 /$ $|j-k(x)-2|^{2}$. Следовательно,

$$
\sum_{j=k(x)+a+2}^{N+1}\left|\delta_{j}(x)\right| \leqslant \sum_{j=k(x)+a+2}^{N+1} \frac{40}{|j-k(x)-2|^{2}} \leqslant 40 \sum_{s=a}^{\infty} \frac{1}{s^{2}}<10 .
$$

Отсюда (см. (14)) получаем

$$
\left|F_{N+1}(x)\right| \leqslant \alpha \sqrt{N}+70 a+80 \leqslant \alpha \sqrt{W}+70 W^{\widetilde{\varepsilon}}+80 \leqslant(\alpha+150) W^{\widetilde{\varepsilon}} .
$$

Таким образом, в обоих случаях (см. (13), (17)) для всех $x \in[0,2 \pi)$ выполнено $\left|F_{N+1}(x)\right| \leqslant$ $(\alpha+150) W^{\widetilde{\varepsilon}}$. Следовательно,

$$
\left\|p_{n}\right\|_{1} \geqslant \frac{1}{3}, \quad\left\|p_{n}\right\|_{\infty} \leqslant 70, \quad\left\|F_{n}\right\|_{\infty} \leqslant(\alpha+150) W^{\widetilde{\varepsilon}}, \quad n=k_{0}+a+1, \ldots, W .
$$

Из (18) нетрудно получить, что неравенство (1) выполнено с константой $C=8 k_{0}+\alpha+150$ для произвольного $W$ (где для $W<8 k_{0}$ в качестве полиномов $p_{k}(x)$ мы положим $p_{k}(x) \equiv 1$, $k=1, \ldots, W)$. Теорема 2 доказана.

\section{СПИСОК ЦИТИРОВАННОЙ ЛИТЕРАТУРЫ}

[1] А. Зигмунд, Тригонометрические ряды, Т. 1, 2, Мир, М., 1965. [2] Б. С. Кашин, В.Н. Темляков, Метрическая теория функиий и смежные вопросы анализа, Сборник статей. Посвящается Петру Лаврентьевичу Ульянову к его семидесятилетию, АФЦ, М., 1999, 69-99. [3] Б. С. Кашин, В.Н.Темляков, Матем. заметки, 64:4 (1998), 637-640. [4] А. О. Радомский, Матем. заметки, 89:4 (2011), 589-595. [5] П. Г. Григорьев, Матем. заметки, 61:6 (1997), 935-938.

\section{А. О. Радомский}

Поступило

Московский государственный

17.06 .2013

университет им. М. В. Ломоносова

E-mail: artrad@list.ru 